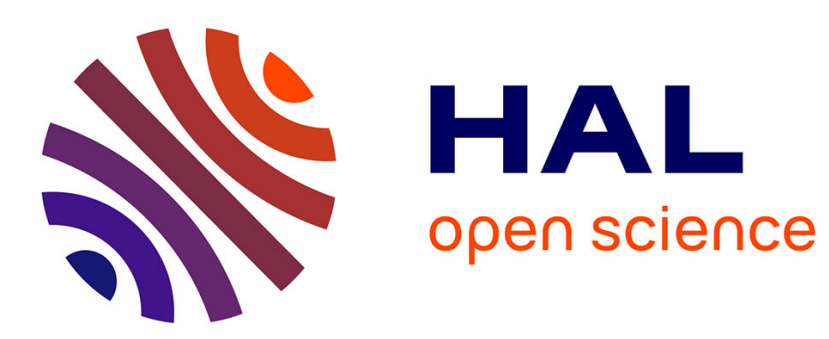

\title{
Complémentarité solaire - électricité
}

\author{
M. Schneider, X. Berger
}

\section{To cite this version:}

M. Schneider, X. Berger. Complémentarité solaire - électricité. Revue de Physique Appliquée, 1982,

17 (9), pp.531-539. 10.1051/rphysap:01982001709053100 . jpa-00245030

\section{HAL Id: jpa-00245030 https://hal.science/jpa-00245030}

Submitted on 1 Jan 1982

HAL is a multi-disciplinary open access archive for the deposit and dissemination of scientific research documents, whether they are published or not. The documents may come from teaching and research institutions in France or abroad, or from public or private research centers.
L'archive ouverte pluridisciplinaire HAL, est destinée au dépôt et à la diffusion de documents scientifiques de niveau recherche, publiés ou non, émanant des établissements d'enseignement et de recherche français ou étrangers, des laboratoires publics ou privés. 
Classification

Physics Abstracts

$86.10 \mathrm{~K}$

\title{
Complémentarité solaire - électricité
}

\author{
M. Schneider et X. Berger \\ C.N.R.R.S., Groupe Ecothermique Solaire, Centre de Recherches Bâtiments Solaires C.N.R.S.-C.S.T.B., \\ Sophia-Antipolis, BP 21, 06562 Valbonne cedex, France
}

(Reçu le 22 octobre 1981, révisé le 27 janvier 1982, accepté le 8 février 1982)

\begin{abstract}
Résumé. - Partant du principe qu'un chauffage d'appoint est nécessaire à tout chauffage climatique, l'optimum financier pour l'usager doit tenir compte des spécificités propres aux deux systèmes. Cet optimum est ici recherché pour le cas d'un appoint électrique. Différentes solutions économiques et intéressantes sont discutées. Une réalisation en cours, basée sur l'une d'entre elles est finalement décrite.
\end{abstract}

\begin{abstract}
Considering the necessity of a power supply to any climatic heating system, the financial optimal solution for the user must take into account the specifies of each system. This optimum is here sought for the case of an electric heating. Different economic and interesting solutions are discussed. At the end, a house, using one of the proposed solutions, is described.
\end{abstract}

1. Introduction. - Un système solaire de chauffage, si tentant qu'il soit quant aux économies qu'il permet de réaliser, et quant à l'assurance d'un certain confort qu'il peut procurer, nécessite un chauffage d'appoint. De nombreuses études de stratégie d'emploi portant sur des systèmes climatiques (solaire, éolien, pompe à chaleur) ont déjà été publiées. Ces études avaient pour but de montrer, soit la complémentarité de deux sources climatiques [1,11], soit l'importance de l'investissement primaire à réaliser en fonction de la couverture des besoins [2,3], sans trop se soucier d'optimiser l'appoint selon ses caractéristiques propres.

Parmi les appoints possibles, l'énergie électrique présente un certain nombre d'avantages : faible investissement, contrôle thermique rapide de chaque pièce, existence d'un tarif de nuit avantageux. Le but du présent article est de rechercher l'optimum financier d'un système mixte solaire-électrique devant assurer la totalité des besoins, le couplage étant réalisé par un stockage minimal rempli alternativement par les deux sources d'énergie afin d'assurer les besoins en début et fin de nuit. Une véritable complémentarité ne peut exister que si les apports solaires journaliers sont commensurables avec l'énergie électrique qui doit être fournie pendant l'ensemble des heures à tarif réduit. Les régions à faible ensoleillement ne peuvent bénéficier que de l'association stockage-chauffage électrique ce qui n'exclut pas la possibilité de réaliser une part importante d'économie par l'emploi d'énergie solaire (techniques passives).
Un modèle de simulation a été construit qui tient compte de la structure tarifaire actuelle et des données climatologiques. Ce modèle conduit à plusieurs solutions économiquement intéressantes qui sont discutées. Une réalisation en cours, basée sur l'une de ces solutions, est finalement présentée.

2. Caractéristiques du programme de simulation. La recherche d'un optimum économique, conduit nécessairement à des captages et stockages minima de l'ordre d'une fraction de jour.

Les séquences de temps jouent donc un rôle mineur et l'élément essentiel est la structure temporelle journalière des apports et des besoins en fonction de la saison et des types de temps.

Les solutions architecturales adaptées à ces stockages de quelques heures sont naturellement données par l'emploi de structures intégrées à l'habitat : murs de refends, dalles...

2.1 La STRUCTURE TARIFAIRE EDF. - Il existe plusieurs sortes de tarifs; le tarif jour-nuit, commun à la plupart des usagers, est le seul considéré dans cette étude. A ce tarif de base se superpose pendant les périodes d'hiver, pour certains gros consommateurs, un tarif de pointe, actuellement stabilisé à heures fixes, mais qui pourrait dans un avenir proche être programmé sur préavis par EDF dans la limite de $400 \mathrm{~h} / \mathrm{an}$ [12]. Le prix voisinerait le décuple du tarif de base. Un stockage au niveau de l'habitat permettrait d'éviter ces heures de pointe dans la plupart des cas. 
Pour les systèmes utilisant une pompe à chaleur soumise à des variations d'apports climatiques (p.a.c. sur l'air extérieur ou associée à des capteurs climatiques ([4]), la présence d'un stockage permet de fonctionner même par température extérieure très froide. L'optimisation de tels systèmes comprend le choix du type de stockage (froid, tiède ou chaud, à chaleur sensible ou à chaleur latente, à stratification ou non), taille du stockage, heures préférentielles de remplissage en fonction de l'enthalpie de l'air au cours de la journée, stratégie de gestion en fonction des séquences de temps types. Elle fera l'objet d'un article ultérieur.

Il n'est ici question que des systèmes électriques utilisant des résistances ou des p.a.c. n'ayant aucune contrainte climatique de fonctionnement (p.a.c. sur nappe phréatique ou en reprise sur l'air extrait; adoption d'un COP moyen hivernal).

Le tarif de nuit est établi entre $23 \mathrm{~h}$ et $7 \mathrm{~h}$, à une heure près, fonction des secteurs géographiques. Le prix du $\mathrm{kWh}$ nocturne est actuellement 0,58 fois le tarif de base. Par ailleurs les factures électriques comportent une part abonnement dont le montant est lié à la puissance installée : $3 \mathrm{kWh}$ de puissance installée supplémentaire coûtant aujourd'hui $400 \mathrm{~F}$ de plus par an.

2.2 Considérations MÉTÉOROLOGiQues. - Les apports solaires sont fonction du rayonnement global incident et de la température de l'air extérieur (Fig. 1). [Pour les capteurs climatiques (à ruissellement ou à condensation) il y a dépendance avec l'hygrométrie. Ces capteurs étant encore au stade expérimental, ce paramètre n'a pas été introduit.] Les apports solaires hivernaux peuvent donc être considérés comme étalés en hiver entre $11 \mathrm{~h}$ et $17 \mathrm{~h}$. La complémentarité dépend principalement de la manière dont le chauffage est assuré pendant les deux périodes critiques $17 \mathrm{~h}-23 \mathrm{~h}$ et $7 \mathrm{~h}-11 \mathrm{~h}$. C'est pourquoi les régions concernées sont celles à jours d'ensoleillement nombreux et à températures douces.

La région niçoise se prête particulièrement bien à une recherche sur de tels systèmes complémentaires; elle est en effet partagée entre deux types de temps : le ciel clair $(48 \%$ des journées, fraction d'insolation moyenne $\sigma=0,8)$ et le ciel couvert $(\sigma=0)$. Le tableau I indique la répartition des besoins selon les différentes périodes de la journée, le type de temps et l'époque de l'année pour des journées moyennes, l'unité «cs » adoptée étant l'état des besoins par beau temps au 15 janvier. Ces besoins ignorent les apports solaires ou gratuits et ne dérivent que de la relation :

$$
W=G V\left(t_{\text {int }}-t_{\mathrm{ext}}\right)
$$

avec $G$ : coefficient de déperditions volumiques de l'habitat

$V$ : volume de l'habitat

$t_{\text {int }}$ : température intérieure $\left(18^{\circ}\right.$ de $23 \mathrm{~h}$ à $7 \mathrm{~h}$

$t_{\mathrm{ext}}:$ température de l'air extérieur.
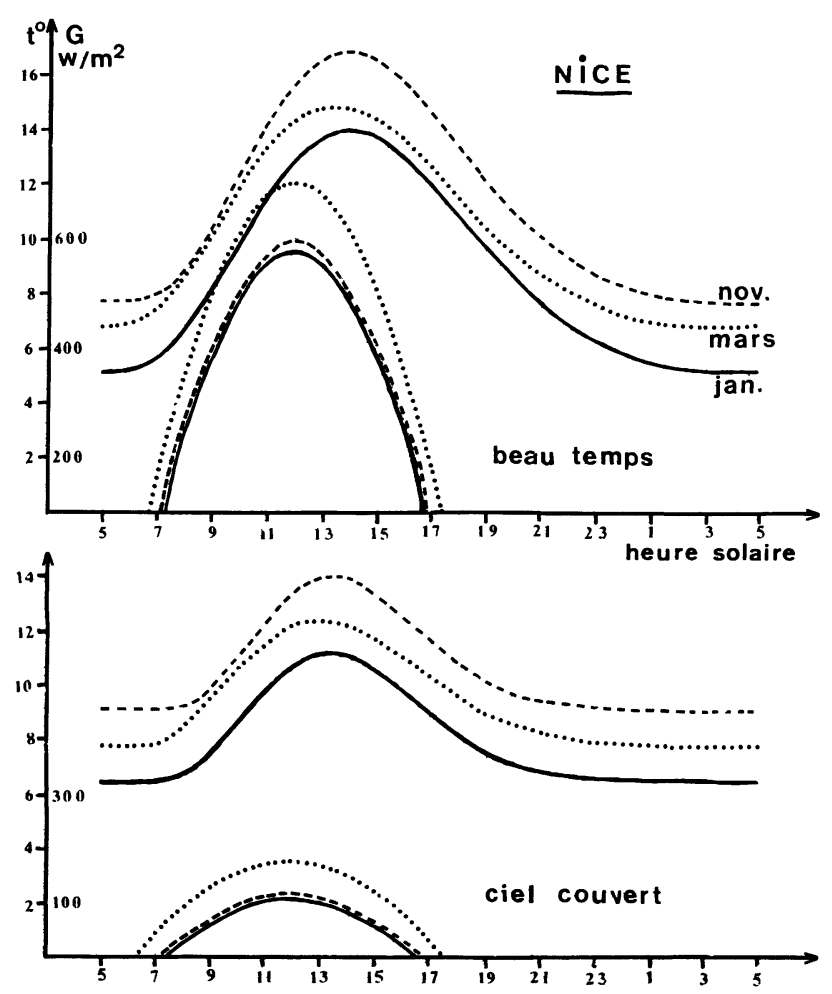

Fig. 1. - Rayonnement global incident sur un plan sud incliné à $45^{\circ}$, et température de l'air extérieur en fonction de l'heure de la journée, du type de temps et de l'époque de l'année.

[Total radiation incident on a south surface inclined at $45^{\circ}$, and external air temperature as functions of the hour of the day, the type of climatic days, and of the epoch of the year.]

$\mathrm{La}$ réduction de $1^{\circ}$ de la température intérieure pendant les $8 \mathrm{~h}$ de nuit est bénéfique. Une valeur plus grande aurait pu être adoptée mais cette réduction permet déjà d'égaliser par beau temps les besoins pendant $8 \mathrm{~h}$ de nuit et les besoins pendant $12 \mathrm{~h}$ de jour.

Le tableau I appelle deux remarques :

a) Par mauvais temps, les besoins diurnes sont importants; le développement des capteurs climatiques est donc de grand intérêt [2-4].

b) Pour un chauffage tout-électrique, le tarif de nuit (sans stockage) permet d'économiser $16 \%$ de la dépense financière de chauffage (en considérant que la différence de coût d'abonnement 1 tranche- 2 tranches est amortie par l'exploitation d'un chauffe-eau électrique à accumulation. S'il n'en est pas ainsi, l'avantage est à peine de $10 \%$ ).

La dépense pour les 12 heures de jour $11 \mathrm{~h}-23 \mathrm{~h}$ représente dans ces conditions $50 \%$ de la dépense totale.

2.3 Algorithme De Calcul. - Le fait d'avoir choisi une valeur normalisée « cs " permet de s'affranchir de la considération d'un système particulier; cette unité est définie par les besoins journaliers de 
Tableau I. - Répartition des besoins de chauffage au cours de la journée à Nice en fonction du type de temps et de lépoque de lannée. L'unité est donnée par les besoins journaliers en janvier par beau temps.

[Distribution of the heating loads during the day in Nice, as a function of the type of climatic days and of the epoch of the year. The unity is defined by the heating loads in january for a clear day.]

\begin{tabular}{|c|c|c|c|c|c|c|c|}
\hline & \multicolumn{2}{|c|}{15 nov } & \multicolumn{2}{|c|}{$15 \mathrm{jan}$} & \multicolumn{2}{|c|}{15 mars } & \multirow{2}{*}{ Total } \\
\hline & $\begin{array}{c}\text { beau } \\
\text { temps }\end{array}$ & $\begin{array}{l}\text { ciel } \\
\text { couvert }\end{array}$ & $\begin{array}{l}\text { beau } \\
\text { temps }\end{array}$ & $\begin{array}{l}\text { ciel } \\
\text { couvert }\end{array}$ & $\begin{array}{l}\text { beau } \\
\text { temps }\end{array}$ & $\begin{array}{l}\text { ciel } \\
\text { couvert }\end{array}$ & \\
\hline $7 \mathrm{~h}$ à $11 \mathrm{~h}$ & 0,15 & 0,15 & $0,19 \mathrm{cs}$ & 0,19 & 0,15 & 0,17 & $\begin{array}{c}1,00 \rightarrow, 22 \% \text { facture } \\
\text { financière }\end{array}$ \\
\hline $11 \mathrm{~h}$ à $23 \mathrm{~h}$ & 0,25 & 0,36 & $0,39 \mathrm{cs}$ & 0,49 & 0,34 & 0,42 & $\begin{array}{c}2,25+50 \% \text { facture } \\
\text { financière }\end{array}$ \\
\hline $23 \mathrm{~h}$ à $7 \mathrm{~h}$ & 0.33 & 0,29 & $0,42 \mathrm{cs}$ & 0,37 & 0,36 & 0,33 & $2,10 \rightarrow \times 0,58=1,22$ \\
\hline Total & 0,73 & 0,80 & 1 & 1,05 & 0,85 & 0,92 & 4,47 \\
\hline
\end{tabular}

l'habitation par $8,5^{\circ}$ de température moyenne de l'air extérieur, en ignorant les apports directs ou gratuits. Elle inclut l'énergie secondaire (électricité...) nécessaire au fonctionnement des capteurs (on a adopté ici $5 \%$ de l'énergie captée) ou à la restitution du stockage [2].

Deux cas ont été considérés :

- celui d'une inertie faible pour lequel les apports solaires sont répartis de $11 \mathrm{~h}$ à $17 \mathrm{~h}$.

- celui d'une inertie forte pour lequel ils sont étalés de $11 \mathrm{~h}$ à $23 \mathrm{~h}$.

Sans stockage, ces apports excédentaires ne sont pas disponibles en dehors de ces séquences de temps.

Conventionnellement on a admis que le captage est proportionnel à l'intensité du rayonnement incident sur un plan incliné à $45^{\circ}$ vers le sud. Pour une fraction d'insolation nulle (temps couvert) on a adopté un captage égal à $20 \%$ de celui obtenu par ciel clair. On tient ainsi compte raisonnablement des divers modes de captage juxtaposés qui peuvent exister : fenêtres, serre, toiture, capteurs... Les besoins de l'habitation ont été calculés heure par heure en fonction de la température de l'air extérieur. Aucune déperdition dans le stockage et pour la restitution n'a été considérée.

Il n'a pas été tenu compte des différents modes de vie des habitants qui peuvent exister, ni des particularités que peuvent avoir les systèmes de chauffage (seuils de fonctionnement, givrages, apports gratuits...), tant elles peuvent être diverses, cela afin de ne pas alourdir la présentation des résultats; une prise en compte peut changer les résultats si les particularités sont importantes. Si la méthode utilisée est restée volontairement simple, c'est pour mieux attirer l'attention sur le dimensionnement optimal du captage et du stockage, et sur l'importance du choix du mode de captage [2].

L'équation générale de fonctionnement du système de chauffage est la suivante :

$$
G V\left(t_{\text {int }}-t_{\text {ext }}\right)-A_{\mathrm{pg}}+\frac{\mathrm{d} E_{\text {stock }}}{\mathrm{d} t}=\eta S \varphi_{\odot}+K_{\mathrm{j}-\mathrm{n}} W_{\text {elect }}
$$

avec :

$A_{\mathrm{pg}}:$ apports gratuits

$\eta$ : coefficient de performance du système de captage

$S:$ surface des capteurs (ou équivalents)

$\varphi_{\odot}$ : rayonnement global incident sur un plan incliné à $45^{\circ}$

$\frac{\mathrm{d} E_{\text {stock }}}{\mathrm{d} t}$ : énergie stockée $(>0)$ ou destockée $(<0)$

$K_{\mathrm{j}-\mathrm{n}}$ : rapport des tarifs électriques «jour» et «nuit»

$W_{\text {elect }}$ : énergie électrique dépensée pour satisfaire les besoins immédiats et, pendant la nuit, pour assurer le stockage nécessaire pour les besoins du lendemain.

$W_{\text {elect }}$ est fonction de la puissance installée de la puissance de stockage maximale de la durée de conservation du stockage, des besoins du lendemain.

Les besoins du lendemain sont fonction de la distribution probabiliste des types de temps de l'époque de l'année.

La consommation de l'appoint électrique a été fixée de manière optimale pour répondre aux besoins de stockage du jour correspondant en admettant une prévision infaillible du type de temps du jour suivant. Les modélisations effectuées avec une prévision selon la distribution des séquences de temps réf. [2] 
donnent une augmentation de la dépense financière de 2 à $5 \%$ pour les systèmes comprenant un stockage.

On peut également fonder la stratégie de stockage sur les prévisions météorologiques à $24 \mathrm{~h}$ qui ont une fiabilité de l'ordre de $80 \%$. La dégradation dans ce cas peut rester dans les limites de 1 à $2 \%$.

3. Analyse des résultats. - Pour la consommation électrique seule, les résultats sont portés sur la figure 2 . Quelques remarques générales sont à faire : l'intérêt du tarif de nuit est d'autant plus grand qu'il y a effectivement consommation de courant au tarif de nuit; ce tarif est donc d'autant plus justifiée s'il existe un stockage et si l'on ne dispose pas d'une pompe à cha-

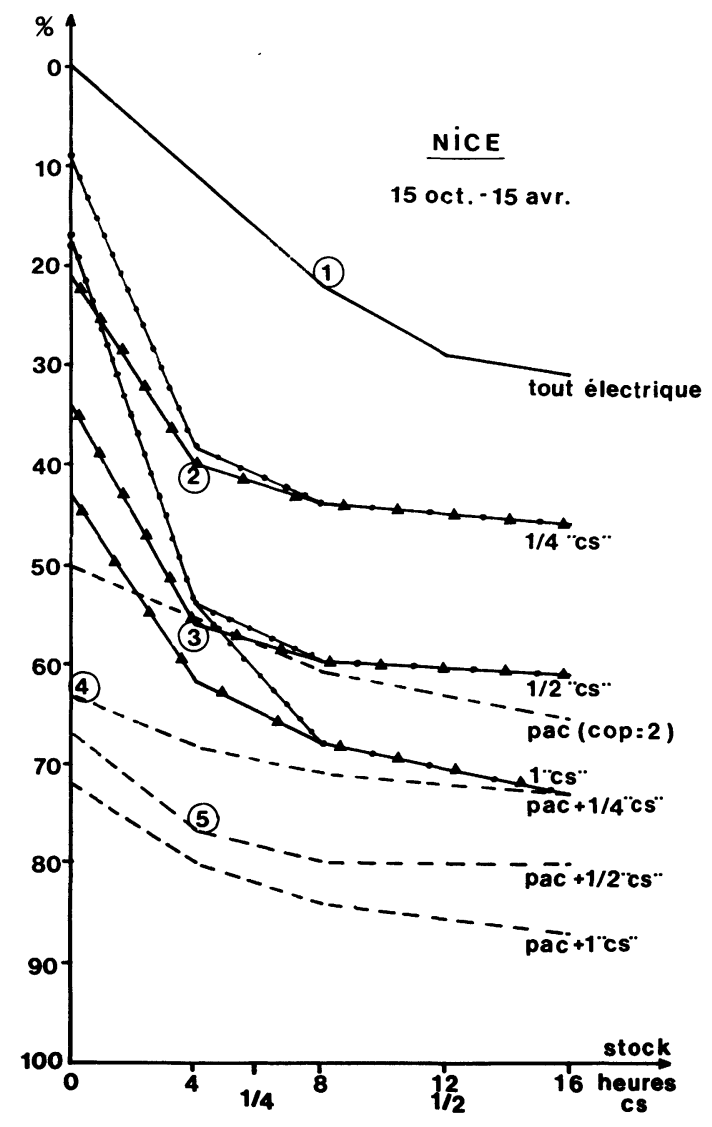

Fig. 2. - Pourcentage d'économie par rapport au chauffage tout électrique, en fonction de la puissance de stockage $(12 \mathrm{~h}=1 / 2$ "cs "), de la puissance de captage, et de la présence d'une pompe à chaleur. "cs " = besoins pour $t_{\text {ext }}=8,5^{\circ} \mathrm{C}$. Les chiffres encerclés correspondent aux solutions optimales. $\mathbf{\Delta}-\mathbf{\Delta}-\boldsymbol{\Delta}$ apports solaires étalés de $11 \mathrm{~h}$ à $23 \mathrm{~h}$. - apports solaires étalés de $11 \mathrm{~h}$ à $17 \mathrm{~h}$. - - - - captage par pompe à chaleur.

[Percent of economy relatively to a completely electric heating, as a function of the storage power, the collecting power, and the existence of a heating pump. " cs " = heating loads for $t_{\text {ext }}=8.5^{\circ} \mathrm{C}$. The encircled numbers are reffered to the optimal solutions. $\Delta-\Delta-$ solar gains displaied from $11 \mathrm{~h}$ to $23 \mathrm{~h}$. $\longrightarrow-$ solar gains displaied from $11 \mathrm{~h}$ to $17 \mathrm{~h}$. $\ldots$ pump.] leur. Dans ce cas il existe une solution optimale pour laquelle la complémentarité solaire-électricité est la mieux établie : captage pas trop important; stockage à remplir parfois par l'électricité, parfois par les apports solaires.

Si la consommation électrique nocturne tombe au-dessous d'un certain seuil, de l'ordre de $1500 \mathrm{kWh} /$ an, l'utilisation d'un tarif à deux tranches ne se justifie plus. L'intérêt d'un grand stockage $(16 \mathrm{~h}$ ou $2 / 3$ «cs ») est minime; le stockage le plus important est associé à une installation sans apports solaires ni pompe : 0,47 «cs »; mais pour d'autres raisons que nous détaillerons plus loin, la taille optimale est moindre.

L'intérêt du stockage dépend de l'importance du captage : un captage trop faible entraîne le remplissage uniquement par l'électricité ; un captage fort nécessite un stockage pour le déphasage dans le temps des apports; si le stockage se dégrade rapidement, un tel captage n'est utile qu'en milieu d'hiver. Un stockage qui ne se détériore pas dans le temps n'a d'intérêt qu'avec un captage important [2].

Cinq solutions, notées de 1 à 5 sur la figure 2 se présentent a priori comme les plus intéressantes; l'analyse de chacune mérite d'être faite et permet des comparaisons entre elles; les commentaires à tirer pour les autres s'en déduisent implicitement :

1) Solution "tout électrique »; pas de système solaire; association d'un chauffage électrique et d'un stockage égale à $1 / 3$ «cs ». Cette solution fait économiser $22 \%$ de la dépense financière. Le stockage est rempli par utilisation de courant électrique toutes les nuits pendant tout l'hiver.

Considérons l'exemple d'une installation pour laquelle la dépense en courant électrique de chauffage revient à $3000 \mathrm{~F}$ (《 cs » $\simeq 100 \mathrm{kWh}$ ), consommation seule hors abonnement. Le prix d'investissement d'un petit stockage (<1《cs ») peut être estimé à $300 \mathrm{~F}$ par $\mathrm{kWh}$. Pour les 15 premiers $\mathrm{kWh}$ l'utilisation naturelle des parois ou dalles peut abaisser ce prix à une constante égale à $1000 \mathrm{~F}$ (surcoût d'adaptation); on a ainsi :

$$
\begin{aligned}
\text { prix du stock }= & 1000 \mathrm{~F}+ \\
& +(\text { stock en } « \mathrm{cs} »-0,15) \times 30000 \mathrm{~F}
\end{aligned}
$$

L'économie annuelle réalisée sur la consommation est de $660 \mathrm{~F}$. Mais pour pouvoir remplir le stockage et assurer en même temps les besoins nocturnes, il faut une surpuissance d'abonnement de $4,8 \mathrm{~kW}$ qui peut être limitée à $3 \mathrm{~kW}$ si on évite de faire fonctionner de nuit certains appareils ménagers (machines à laver...). La taxe fixe d'abonnement est majorée annuellement de $400 \mathrm{~F}$, ce qui entraîne un amortissement en 25 ans.

Un stockage de $1 / 2$ "cs » au lieu de $1 / 3$ ferait économiser $900 \mathrm{~F}$ de consommation, mais nécessiterait une surpuissance installée d'au moins $6 \mathrm{~kW}$. Compte tenu d'une nouvelle augmentation de l'abonnement, l'économie tomberait à $100 \mathrm{~F}$ par an (Fig. 3). 


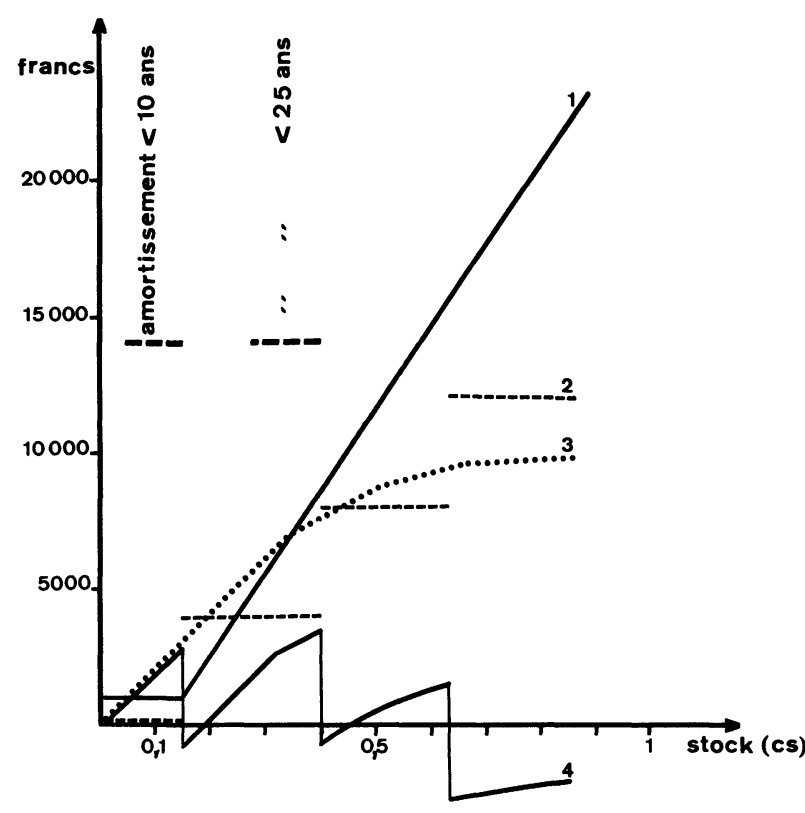

Fig. 3. - Rentabilité de la solution tout électrique. 1. Coût d'installation du stockage. 2. Dépense d'abonnement cumulée sur 10 ans. 3. Economie de consommation cumulée sur 10 ans. 4. Economie nette de consommation cumulée sur 10 ans.

[Efficiency of the completely electric solution. 1. Set-up cost of the storage. 2. Subscription cost for 10 years. 3. Economy of consumption for 10 years. 4. Net economy of consumption for 10 years.]

Cette discussion fait bien apparaître l'incidence du coût des surpuissinces installées. Il y aurait sans doute intérêt pour EDF, dans la limite des puissances acceptables par le réseau de distribution, à instituer un abonnement à deux niveaux de puissance, jour et nuit.

Même sans modifier la structure actuelle des abonnements, du fait que la majorité des usagers n'utilise pas à plein les puissances d'abonnement souscrites, il y a sans doute intérêt à installer un petit stockage $(0,15$ « cs ») rempli au tarif de nuit pendant les périodes où la puissance maximale n'est pas appelée.

2) captage égal à 0,25 «cs "; stockage égal à 0,15 « cs ». Le captage fait gagner $21 \%$ sur la facture financière, le stockage $19 \%$. La charge électrique du stockage est faite systématiquement par mauvais temps en toutes saisons, et par beau temps seulement en milieu d'hiver; on la limite à $1 / 3$ en début et en fin d'hiver. Le captage solaire associé est de $25 \mathrm{kWh}$ environ, les 15 premiers $\mathrm{kWh}$ peuvent être considérés comme gratuits (ils peuvent en effet être assurés par les fenêtres et les baies vitrées). Les $\mathrm{kWh}$ suivants nécessitent une adaptation (serre accolée, véranda, store, système à chaleur latente...), pour éviter les problèmes d'inconfort. On peut également les obtenir à partir de $5 \mathrm{~m}^{2}$ de capteur à un prix moyen de $5000 \mathrm{~F}$. Dans ces conditions, qui correspondent à une bonne intégration à l'habitat, la solution 2 permet l'amortissement de l'installation en 12 ans environ (cf. Fig. 4).

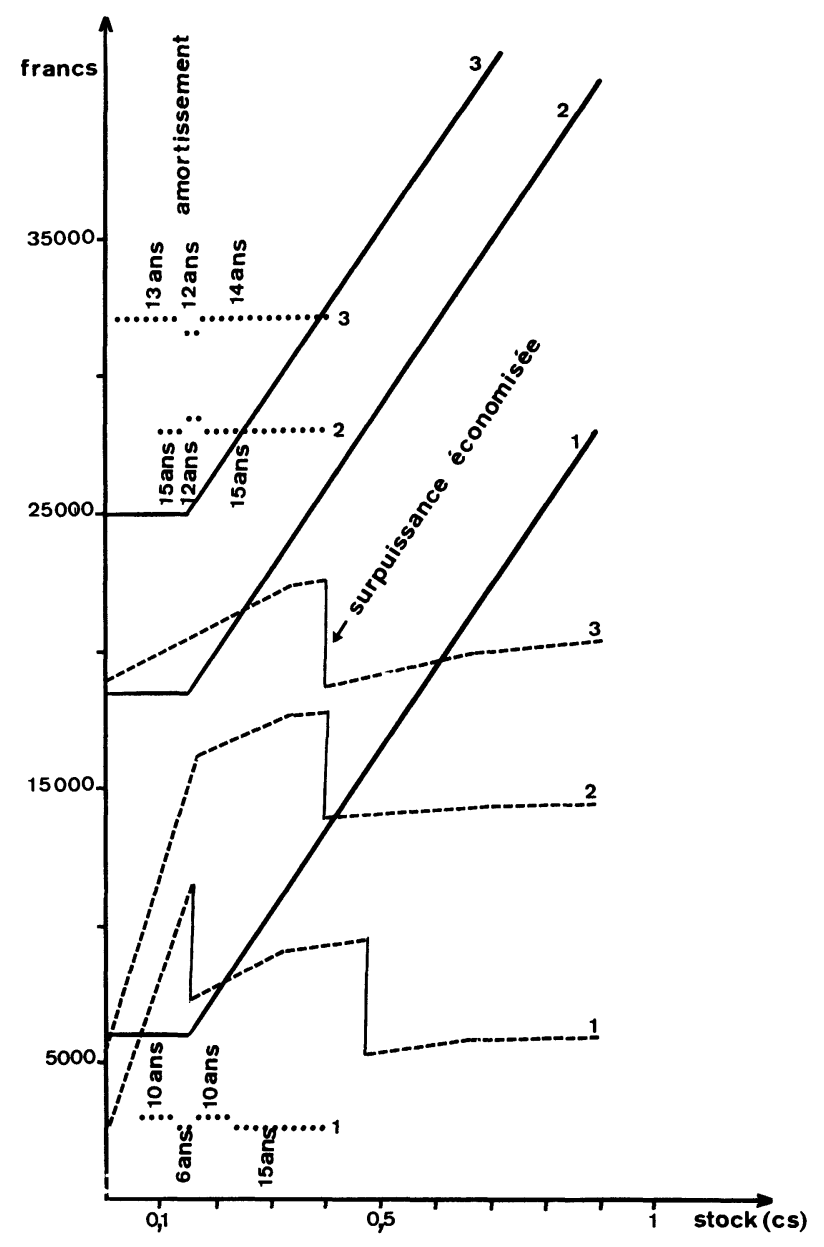

Fig. 4. - Bénéfice net en 10 ans (- - - ) et durée d'amortissement (...) en fonction du coût d'investissement (1. Cas d'un captage égal à 0,25 «cs ». 2. Cas d'un captage égal à 0,50 «cs ». 3. Cas d'une pompe à chaleur sans captage solaire.

[Net profit in 10 years $(--\infty)$ and redemption time (...) as functions of the investment cost (-). 1. Collecting equal to 0.25 «cs». 2. Collecting equal to 0.50 «cs ». 3. Heat pump without solar collecting.]

Les comparaisons avec les autres cas d'installation, montrent que cette solution est la meilleure de celles qui sont ici recherchées (stockage $=0,15$ "cs", captage $=0,20 \ll c s »)$.

3) Captage égal à 0,5 «cs»; stockage égal à 0,15 «cs » ou $4 \mathrm{~h}$ des besoins de chauffage en janvier.

Le captage fait économiser $34 \%$ sur le bilan financier de chauffage, le stockage $22 \%$. Le stockage est rempli par utilisation de courant électrique à raison de $15 \%$ par beau temps en milieu d'hiver, et complètement en toutes saisons par temps couvert. Les apports solaires assurent un taux de remplissage important et la solution est bien dimensionnée; si le captage s'amortit moins bien que pour la solution 2, puisqu'il n'engendre pas une économie double, le stockage peut, par contre, dans les conditions de la solution 1, être amorti en 13 ans, s'il n'y a pas de problème de surpuissance installée; un tel problème se pose d'ailleurs 
faiblement, compte tenu du peu de jours où la surpuissance installée serait utilisée.

4) Pas de stockage, mais association d'une pompe à chaleur et d'un captage de 0,25 " cs ».

L'intérêt de cette solution est d'associer un capteur et une pompe à chaleur pour diminuer la taille du captage (récupération des basses calories), sa sophistication (capteurs sans vitrage), éviter les problèmes de givrage de la pompe, et faire fonctionner la pompe avec un excellent COP. Il faut compter que ce n'est pas $13 \%$ d'économie que le captage apporte, mais 3 fois plus si l'on tient compte de ce que l'on gagne sur le fonctionnement de la pompe. Avec un investissement estimé à $24000 \mathrm{~F}$ pour la p.a.c., la solution 4 présente un amortissement moins bon que la solution 3. Ce qui montre l'attention qu'il faut porter à l'association de deux systèmes qui se recoupent partiellement et dont les performances ne sont pas additives.

5) Association d'une pompe à chaleur, d'un captage de 0,5 « cs » et d'un stockage de 0,15 « cs ».

L'interaction captage-stockage-pompe-électricité, est très grande : le stockage n'a d'intérêt que si le captage est suffisant ; l'importance du captage diminue l'intérêt de la pompe, mais celle-ci retrouve son avantage par la présence d'un stockage. En effet, l'économie est réalisée presque entièrement sur la consommation pendant les jours de mauvais temps. Cette solution très performante sur le plan énergétique ne présente aucune rentabilité financière.

4. Application : les maisons solaires de Biot. 4.1 DeSCRIPTION. - Deux maisons en construction à Biot (Alpes-Maritimes) illustrent la solution 3 explicitée ci-dessus. Elles sont réalisées dans un lotissement de 36 maisons du même genre, qui comportent des expériences d'économies d'énergie réalisées sur d'autres maisons, expérimentation EDF et GDF.

La forme architecturale est imposée pour l'ensemble du lotissement. (Architecte G. Gratien, 06 Antibes.)

La solution proposée recherche un optimum économique, tant du point de vue investissement, que dépense de chauffage, afin de permettre une promotion des systèmes solaires.

Les maisons ont des toitures à une pente, avec terrasse non accessible au-dessus de la salle à manger (ce qui permet l'installation de $4 \mathrm{~m}^{2}$ de capteurs, non visibles, pour l'obtention d'eau chaude sanitaire). La liaison avec le cellier pour l'emplacement du cumulus de stockage d'eau chaude sanitaire est des plus aisées (Fig. 5).

La surface habitable est de $84 \mathrm{~m}^{2}$, le volume $270 \mathrm{~m}^{3}$, le coefficient de déperditions volumiques $G$ vaut $1,14 \mathrm{~W} / \mathrm{m}^{3}{ }^{\circ} \mathrm{C}$.

D'où :

"cs " $\simeq 1,14 \times 270 \times(18,5-8,5) 24=74 \mathrm{kWh}$.

Pour allier efficacité confort et esthétique le système solaire a été composé de deux parties (Fig. 5).
- Une serre accolée avec toiture non vitrée afin d'éviter surchauffe et rayonnement trop intense, en particulier à $1,50 \mathrm{~m}$ de hauteur [5]. Pendant la journée, l'air chaud est envoyé dans la maison par les ouvertures serre-habitat, notamment une fenêtre haute et étroite, donnant sur le séjour, et qui a été ajoutée au plan d'origine. La nuit la serre constitue un espace tampon.

- Un mur de type «Trombe » dont la forme en T pour un stockage et une restitution efficace des calories, provient des plans d'origine de la maison. Pour obtenir un meilleur captage, on a placé en insolation directe, 45 tubes de chliarolithe (diamètre $9 \mathrm{~cm}$, hauteur $2 \mathrm{~m}$, soit au total une surface développée de captage convectif de $14 \mathrm{~m}^{2}$ ). (La chliarolithe est un matériau à chaleur latente de fusion, commercialisé par la société Solvay, sur licence ANVAR-C.N.R.S. [6]).

Une porte vitrée au séjour et une fenêtre à la chambre constituent avec la serre les baies vitrées sud. Les maisons sont juxtaposées et n'ont donc ni ouvertures, ni déperditions sur les faces est et ouest.

Les tubes de chliarolithe n'ont pas été encastrés dans le mur et l'air peut circuler librement entre l'avant des tubes et l'arrière. L'air échauffé sur les tubes en face sud, peut échauffer par convection la face arrière des tubes et le mur en béton, tant du côté sud que sur la partie du « $T$ » intérieure à la maison $\left(50 \mathrm{~m}^{2}\right.$ de surface interne pour les échanges convectifs).

Reprenant l'étude effectuée pour la maison solaire de Grimaud [7], on a disposé un vitrage sélectif Horti + (couche sélective vers l'intérieur du mur) en regard des tubes. Un vitrage ordinaire, en avant du vitrage sélectif, complète la paroi et permet la récupération des calories perdues. La circulation de l'air neuf passe en effet entre les deux vitrages et réalise une isolation dynamique. L'évacuation d'air vicié est maintenue comme dans le plan général de toutes les maisons : reprise par gaine en W.C., salle de bain, cuisine et éjection directe après centralisation en partie nord.

L'air chaud, à l'intérieur du mur, est distribué par une courte gaine sous plafond vers les chambres et le séjour, la reprise se faisant en partie basse par des ouvertures dans le mur sud. Un petit ventilateur $(150 \mathrm{~W})$ réglé par thermostat, réalise la circulation d'air $(1 \mathrm{~V} / \mathrm{h})$.

Des résistances électriques $(8 \mathrm{~kW})$ sont enfin disposées au pied du mur sud. Le chauffage nocturne des pièces est fait avec priorité au mur Trombe et à ses résistances électriques afin de permettre le stockage des calories au tarif " nuit».

4.2 Fonctionnement. Performances. - Les apports directs par les baies vitrées sud et la serre permettent un échauffement rapide de la maison le matin, tandis que le mur Trombe monte lentement en température.

Pendant la journée, si la température de la maison n'est pas suffisante, le ventilateur chauffe la maison en épuisant les calories stockées dans le mur Trombe. Les 


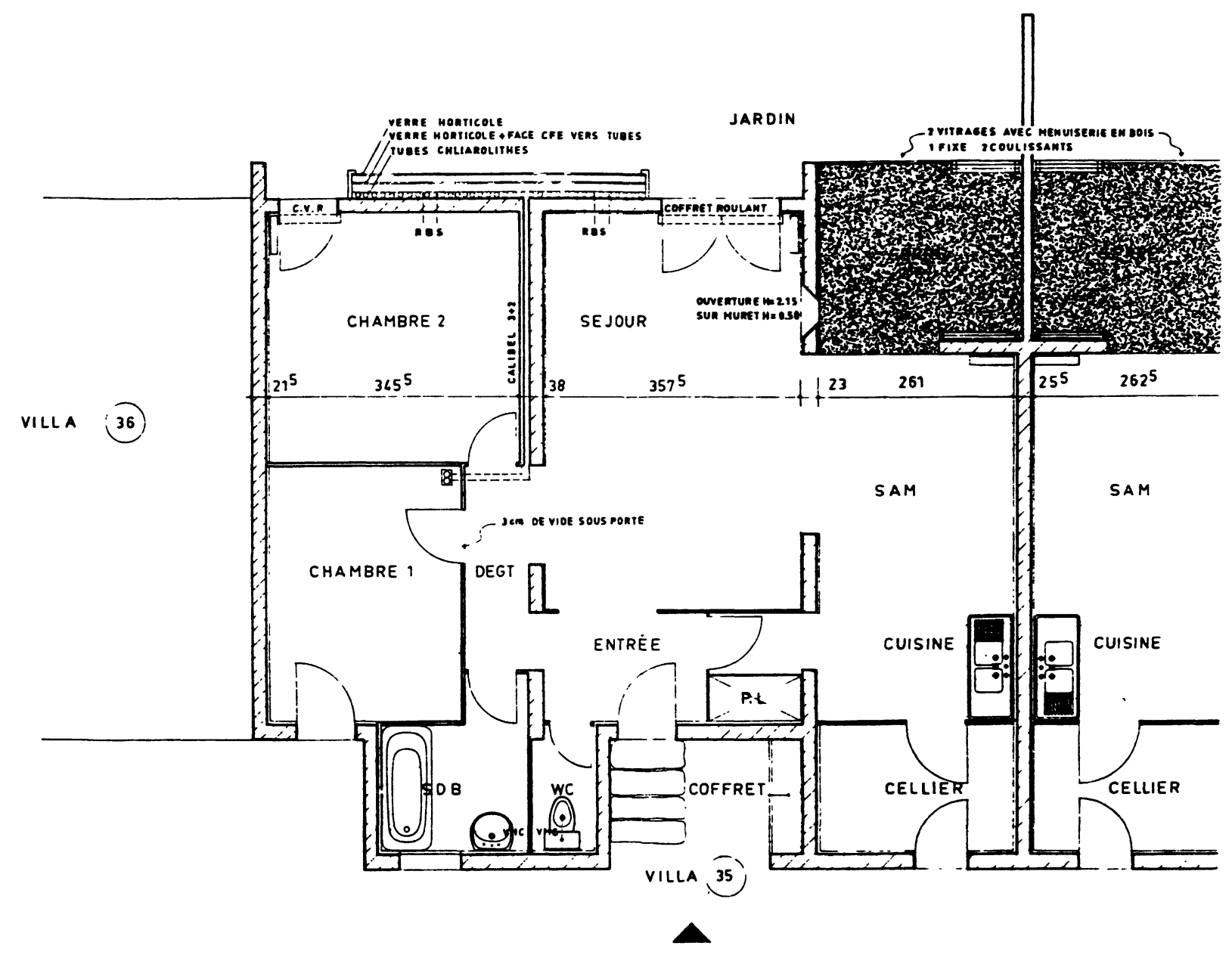

Fig. 5. - Plan des maisons solaires de Biot.

[Draft of the Biot solar houses.]

résistances électriques du mur ne sont mises en route que pendant les heures de tarif de nuit.

Le déphasage des apports thermiques à travers le mur est d'environ 5 heures. L'air neuf, préchauffé par les déperditions du vitrage Horti $+\left(+2^{\circ}\right)$, permet d'apporter près de $5 \mathrm{kWh}$ par jour de beau temps en janvier. Sur les $18 \mathrm{kWh}$ qui parviennent à être captés et stockés par le mur en un tel jour, 3,5 sont donnés à la maison par transmission à travers la paroi (température maximale côté intérieur égale à $24^{\circ}$ ). Le reste est distribué par la ventilation (échauffement de l'air qui circule dans le mur de $6^{\circ}$ en moyenne).

L'efficacité de fonctionnement de ce système, rapport entre l'énergie donnée à la maison et l'énergie solaire incidente, voisine $65 \%$.

La serre et les baies vitrées sud représentent $10 \mathrm{~m}^{2}$ de vitrage, ce qui, compte tenu des occultations par les parois, de la présence de voilages et de poussières [8], correspond, en janvier, à un apport de $20 \mathrm{kWh}$ environ.

Si la puissance de captage globale correspond à 0,55 « cs », celle de stockage est de 0,22 «cs » car le système associé au stockage ne fonctionne pas avec un rendement maximum en raison de sa situation extérieure. On ne peut donc prétendre à une économie de chauffage supérieure à $50 \%$.

$\mathrm{Si}$ l'on considère la couverture des besoins en $\mathrm{kWh}$ [2], le système proposé reste de performances modestes, car il ne permettra de satisfaire que $25 \%$ des besoins. Mais si l'on considère la facture financière, l'économie réalisée atteint $40 \%$ par rapport à une maison identique dépourvue de système solaire et chauffée électriquement. Ceci est encourageant pour plusieurs raisons :

- L'installation du système solaire n'a pas exigé de modifications importantes de la maison. La maison n'a pas été conçue en fonction de lui, mais il s'est adapté à elle.

- Sur le plan esthétique, la facade sud ne se présente pas comme totalement noire. Certes, le mur Trombe a un aspect sombre et irrisé, mais sa taille étant modeste $\left(9 \mathrm{~m}^{2}\right)$, quelques lignes permettent de lui donner un aspect agréable.

- Le surcoût de la serre devrait être compté relativement à son taux d'habitabilité. Ouverte en été, elle constituera une pergola à l'abri du soleil. En hiver, elle sera confortable environ la moitié du temps. 
Finalement, elle sera habitable plus des $3 / 4$ du temps annuel.

C'est donc seulement $25 \%$ de son surcoût qu'il convient de compter comme surcoût solaire (prolongement de la toiture, percée d'une fenêtre étroite avec protection anti-effraction, baie vitrée sud, demicloison commune avec la serre de la maison voisine). Pour le surcoût du mur Trombe, l'isolation interne du mur est transportée au niveau de la partie en «T». Il faut donc seulement compter la gaine de distribution d'air chaud, la gaine non isolée de distribution d'air neuf, le vitrage Horti + et le vitrage ordinaire avec leur châssis, ainsi qu'un ventilateur et 45 tubes de chliarolithe.

Le surcoût solaire peut être estimé à $16000 \mathrm{~F}$, ce qui donne un amortissement en 12 ans. Si la maison devait être réalisée en petite série, on pourrait normalement aboutir à un amortissement en 8 à 10 ans. Néanmoins, même avec l'amortissement actuel, on peut considérer le système comme justifié, si l'on tient compte de la garantie de chauffage qu'il représente à une époque où l'approvisionnement en combustible peut devenir incertain.

5. Conclusion. - Les résultats obtenus font ressortir que la complémentarité solaire-électricité passe nécessairement par l'intermédiaire d'un stockage et que la solution optimale est la solution 2. Dans les régions moins ensoleillées ou plus froides, les solutions 4 et 5 sont particulièrement adaptées afin de tirer profit des basses calories. L'intégration à l'habitat du captage et du stockage permet un bon amortissement du système en ajoutant le bénéfice de fonctions supplémentaires : parois de l'habitat, volumes habitables complémentaires, espaces-tampon.

La réalisation d'un stockage supplémentaire de faible capacité, intégré à l'habitat, est facilitée par l'utilisation d'un matériau à changement de phase ayant une température de fusion supérieure d'une dizaine de degrés à la température d'ambiance [6]. On bénéficie alors d'une puissance de captage élevée en insolation directe du fait de la forte effusivité de ces matériaux. On limite les gradients de température associés, ce qui permet un meilleur confort. Le stockage important permet enfin une restitution en plusieurs heures qui correspond bien aux cas recherchés en première partie.

Le stockage de type annuel (sans dégradation dans le temps) a son intérêt dans la satisfaction des besoins à certains moments critiques : jours très froids, les deux périodes hivernales de 10 jours de temps couvert; pour l'E.D.F., ces moments sont ceux où tous les usagers sont en même temps gros consommateurs de courant, et il $\mathrm{y}$ a donc des problèmes de surpuissance demandée. Pour l'usager, l'intérêt n'est pas de s'en servir une fois par an en rechargeant le stockage en été, mais de transférer des apports solaires excédentaires (surtout en demi-saison) pour les jours de mauvais temps (heures de jour); ainsi, non seulement il ne sert pas à supprimer le captage, mais plutôt il nécessite un captage assez puissant. Par ailleurs, son amortissement est d'autant meilleur qu'il est rechargé plus souvent pendant l'hiver ce qui peut aussi être fait en utilisant le courant électrique au tarif de nuit. Il est donc adapté aux régions à longues séquences de beau et mauvais temps (régions montagneuses). Son intérêt financier ne peut se situer dans le cadre d'une complémentarité avec un chauffage d'appoint tel que l'électricité que pour diminuer la puissance installée. Cependant, même dans ce cas, on ne peut parler que d'assurance d'une couverture totale des besoins mais non d'une optimisation financière [2].

La prévision de tarifs de "pointe " très onéreux ( $400 \mathrm{~h} / \mathrm{an}$ à $2,30 \mathrm{~F}$ par $\mathrm{kWh}$ ), pour les consommateurs ayant 3 tarifs (immeubles, installations importantes) est un encouragement à posséder un stockage. Une expérience de chauffage du bâtiment du "C.I.O.N. ", à l'Observatoire de Nice, se propose de démontrer cette complémentarité solaire-électricité [10] en faisant intervenir les heures de pointes et les contraintes diverses. Les centrales thermiques pouvant difficilement être régulées en fonction d'une demande irrégulière, une des solutions au problème de la régulation est d'encourager par des tarifs parfois préférentiels et parfois onéreux le stockage de courant chez les usagers. La forme thermique est une des plus naturelles. Cela permet d'éviter la conception de réseaux de distribution surpuissants et la réalisation de gros stockages au niveau des centrales électriques.

Pour ce faire il y a lieu de développer les systèmes de contrôle chez l'usager tels que thermostats, répartiteurs de phases, délesteurs, en même temps que les systèmes de captage et stockage intégrés à l'habitat. Les contraintes existantes avec l'usage des énergies climatiques et des chauffages d'appoint doivent être résolues en même temps dans une optimisation de l'ensemble des deux systèmes de chauffage. Le cas des pompes à chaleur avec leurs seuils de fonctionnement et leur COP variable fera l'objet d'une prochaine étude étant donnée son ampleur. La complémentarité entre deux sources d'énergie, si elle est fonction de la manière dont la consommation est faite, est aussi fonction de la démultiplication des effets qui peuvent être obtenus en même temps que des chevauchements qu'il convient d'éviter. 


\section{Bibliographie}

[1] Joubert, A., Pecheux, J., Etude du comportement d'un système énergétique fonctionnant à partir du couplage des énergies solaires et éoliennes. Revue Phys. Appl. XVI (1981) 397.

[2] Berger, X., Chauffage solaire et stratégies de chauffage. Revue Phys. Appl. 16 (1981) 225.

[3] Peube, J.-L., Pecheux, J., PlancQ, E., Sur les conditions optimales d'utilisation de l'énergie solaire pour le chauffage de l'habitat. Revue Phys. Appl. 15 (1980) 553.

[4] Pelletret, R., Etude du capteur à ruissellement (Rapport de fin d'études. Université de Valenciennes et C.N.R.S. Nice, 1981).

[5] Berger, X., Serres accolées à l'habitat-stores vénitiens à chaleur latente (Rapport Plan Construction 1980 « Maison énergie zéro »).

[6] Schneider, M., Sylvain, J.-F., Jaffrin, A., Berger, X., Bourdeau, L., Brevet-Matériau accumulateur de calories à température constante et applications de ce matériau. No 77.36.216.

[7] Henry, J.-J., Berger, X., La maison solaire de Grimaud. Revue Phys. Appl. 15 (1980) 569.
[8] Anquez, J., Croiset, M., Le Quere, J., Incidence des caractéristiques solaires d'un logement sur ses besoins de chauffage (Cahiers du C.S.T.B. No 203 et $219 ; 1979$ et 1981$)$.

[9] Olivet, J., Hamayon, L., Cabinet Teta, Groupe Ecothermique Solaire, Cabinet Parica, T.S.I., Optimisation d'un modèle d'habitat à double façade ventilée (Rapport Plan Construction 1981).

[10] Bourdeau, M., Projet de modification du chauffage pour le bâtiment du C.I.O.N. à l'Observatoire de Nice (Rapport de fin d'études Ecole Centrale 1979).

[11] Bouton, J., Frelin, G., Missenard, M., Rougerie, G., QUEMENER, Y., Réflexions sur les solutions à énergies complémentaires dans le tertiaire neuf (Journées d'études du Comité Français d'électrothermie, Versailles 1981).

[12] Bernard, P., Les principales caractéristiques des structures tarifaires envisagées pour la basse tension (Journées d'études du Comité Français d'électrothermie, Versailles 1981). 